\title{
Low density InAs quantum dots with control in energy emission and top surface location
}

\author{
P. Alonso-González, D. Fuster, L. González, J. Martín-Sánchez, and Y. González \\ Instituto de Microelectrónica de Madrid (IMM-CNM, CSIC), Isaac Newton, 8 Tres Cantos, \\ Madrid 28760, Spain
}

(Received 11 September 2008; accepted 19 October 2008; published online 4 November 2008)

\begin{abstract}
In this work we extend the droplet epitaxy growth technique to the fabrication of low density InAs quantum dots (QDs) on GaAs (001) substrates with control in size, energy emission, and top surface location. In particular, depending on the amount of InAs material deposited, it has been possible to tune the QD energy emission over a range of 1.12-1.40 eV while keeping constant the nanostructures density at $2 \times 10^{8} \mathrm{~cm}^{-2}$. Moreover, the capping growth process of these QD shows mounding features that permit their spatial identification once embedded by a GaAs capping layer. (C) 2008 American Institute of Physics. [DOI: 10.1063/1.3021070]
\end{abstract}

Semiconductor quantum dots (QDs) show high potential as active elements in the fabrication of devices in the quantum information technology field. ${ }^{1-3}$ Devices as triggered sources of single photons ${ }^{3}$ or entangled photon pairs ${ }^{1}$ have been recently achieved using these nanostructures. Furthermore, by embedding them within a photonic crystal microcavity, particular quantum nature by means of strong coupling related phenomena is starting to be tackled. ${ }^{2}$ An essential requisite in these experiments is to ensure an effective coupling between the cavity modes and the emission of a single QD. In this sense, the fabrication technology requires the formation of nanostructures with simultaneous control of size (emission wavelength) and surface location. ${ }^{2}$

As it is well known, self-assembling processes during epitaxial growth provide random distributions of QD with high optical quality. However, the simultaneous control of density and size is not straightforward. ${ }^{4} \mathrm{~A}$ feasible approach to overcome all these inherent constraints is the use of prepatterned substrates where fabricated motives provide preferential sites for the posterior nucleation of QD. ${ }^{5-8}$ The technological processes involved in these approximations generally cause a decrease in the nanostructures optical emission. This inconvenient is avoided in a natural way if the patterning process is carried out in an in situ context. ${ }^{9-11}$ Recent results have shown the droplet epitaxy growth technique as an in situ lithographic tool for the fabrication of low density nanoholes on GaAs substrates. ${ }^{10,11}$ In particular, these nanoholes act as nucleation centers for the formation of QD showing excellent optical properties at the single nanostructure level. ${ }^{11}$

In this work, we extend this growth procedure in order to tune the QD size (emission wavelength), maintaining the total density of nanostructures at low values of $2 \times 10^{8} \mathrm{~cm}^{-2}$. Furthermore, we also demonstrate a direct correspondence between the surface mounds formed during the capping growth process and the buried QDs which would permit a direct surface localization of one active nanostructure. All together, these results mean an advance in those technologies involved in the fabrication of quantum optoelectronics devices, for which one isolated QD of designed emission wavelength located at a specific site is a necessary requirement.

The experimental procedure starts growing a $0.5 \mu \mathrm{m}$ thick undoped GaAs buffer layer at a growth rate $r_{g}=0.5 \mathrm{ML} / \mathrm{s}, \mathrm{As}_{4}$ beam equivalent pressure (BEP) of
$2 \times 10^{-6}$ Torr, and substrate temperature $T_{s}=580^{\circ} \mathrm{C}$ on GaAs (001) substrates by molecular beam epitaxy (MBE). The root mean square roughness of this surface is typically $0.24 \mathrm{~nm} . T_{s}$ is then decreased to $500{ }^{\circ} \mathrm{C}$ and the nanohole template formation process is performed. As previously reported it consists of a two-step process where initially the $\mathrm{Ga}$ droplets are formed by the periodically opening of $\mathrm{Ga}$ $\left(r_{g} \mathrm{GaAs}=0.5 \mathrm{ML} / \mathrm{s}\right)$ and As shutters, and finally, they are annealed under As atmosphere for 6 min at $\mathrm{BEP}_{\mathrm{As}_{4}}=5$ $\times 10^{-7}$ Torr. $^{11}$

In this work, during this last annealing step, In cell is opened for QD formation inside the nanoholes. Once the desired amount of In is deposited, the process is maintained providing only $\mathrm{As}_{4}$ to complete the total time of $6 \mathrm{~min}$. We varied the opening time of In cell to grow 1.1, 1.3, 1.5, and $1.7 \mathrm{ML}$ of InAs $\left(r_{g}=0.01 \mathrm{ML} / \mathrm{s}\right)$. After this step, the formed nanostructures are finally annealed under $\mathrm{As}_{2}$ flux for $1 \mathrm{~min}$ at $T_{s}=510^{\circ} \mathrm{C}$ and BEP of $5 \times 10^{-7}$ Torr. Atomic force microscopy (AFM) measurements were carried out on these samples for QD topographic information.

For photoluminescence (PL) emission studies, a $85 \mathrm{~nm}$ thick GaAs cap layer is grown at $r_{g}=0.5 \mathrm{ML} / \mathrm{s}$. The initial $15 \mathrm{~nm}$ are grown at $T_{s}=510{ }^{\circ} \mathrm{C}$ under $\mathrm{As}_{2}$ at $\mathrm{BEPAs}_{2}$ changing from $5 \times 10^{-7}$ to $9 \times 10^{-7}$ Torr and the final $70 \mathrm{~nm}$ at typical GaAs growth conditions of $T_{s}=580^{\circ} \mathrm{C}$ and BEPAs $_{4}=2 \times 10^{-6}$ Torr. The PL measurements were performed at $T=30 \mathrm{~K}$ with a standard setup using a frequencydoubled $\mathrm{Nd}$ doped yttrium aluminum garnet laser $\left(\lambda_{\text {exc }}\right.$ $=532 \mathrm{~nm}$ ) as the excitation source with a spot diameter of approximately $200 \mu \mathrm{m}$.

In order to study the cap layer surface morphology and its possible correlation with the buried nanostructures, a similar sample as the above described with 1.4 ML of InAs deposited at the nanoholes was covered by a thinner GaAs cap layer of $25 \mathrm{~nm}$. Similar to the PL samples this cap layer was initially grown $(3 \mathrm{~nm})$ at $T_{s}=510^{\circ} \mathrm{C}$ under $\mathrm{As}_{2}$ at changing $\mathrm{BEPAs}_{2}$ from $5 \times 10^{-7}$ to $9 \times 10^{-7}$ Torr and followed by an eventual $22 \mathrm{~nm}$ thick layer at typical MBE GaAs growth conditions. An identical sample but with the deposition of 1.4 ML of InAs on the top surface was also grown.

The AFM topography in Fig. 1(a) shows the nanoholes template formed after arsenization of Ga droplets without any In deposition. The structures obtained, with a density of 

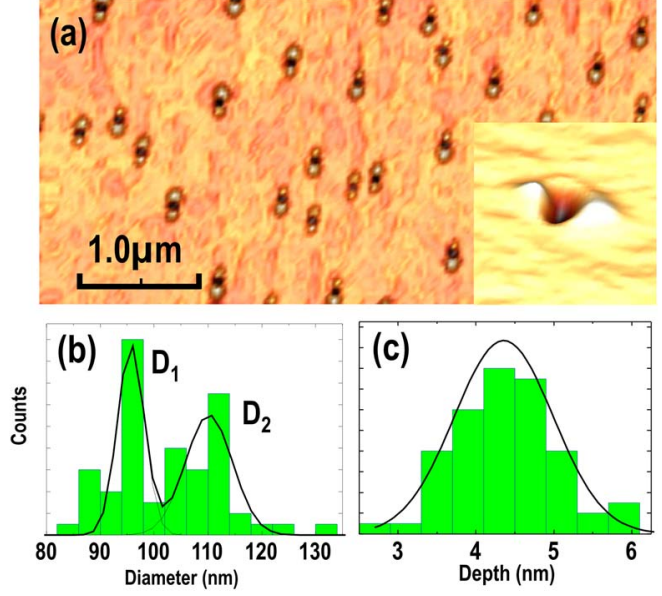

FIG. 1. (Color online) (a) $2.5 \times 5 \mu \mathrm{m}^{2}$ AFM micrograph showing a GaAs nanoholes template performed by droplet epitaxy technique. The inset shows a detailed three-dimensional image of a nanohole. (b) Nanohole template diameter distribution. (c) Nanohole template depth distribution.

$2 \times 10^{8} \mathrm{~cm}^{-2}$, show an elongated structure formed by two lobes that surround a central nanohole. Figures 1(b) and 1(c) show the nanohole diameter and the depth size distribution referred to as the flat surface plane. It can be observed a single distribution, centered at $h=4.4 \pm 0.7 \mathrm{~nm}$, for the nanohole depth and a bimodal distribution, measured by two Gaussian line-shape fit, centered at $D_{1}=95 \pm 5 \mathrm{~nm}$ and $D_{2}=110 \pm 9 \mathrm{~nm}$ for the nanohole diameter. These results suggest an initial Ga droplet bimodal distribution that develops into nanoholes with similar depths but with a clear bimodal distribution of diameters.

The AFM images in Figs. 2(a) and 2(b) show the result of depositing 1.3 and 1.7 ML of InAs on the nanoholes template, respectively. We observe in Fig. 2(a) a bimodal size distribution in the QD nucleated at the nanoholes (labeled in the figure as $\mathrm{QD}_{\mathrm{A}}$ and $\left.\mathrm{QD}_{\mathrm{B}}\right)$. The bimodal distribution previously observed for the diameter of the nanoholes could lead to this situation. Accordingly, the nanoholes with smaller diameters would lead to QD protruding from the nanoholes $\left(\mathrm{QD}_{\mathrm{A}}\right.$ family); while larger nanoholes would lead to QD with a height that hardly surpass the surface level $\left(\mathrm{QD}_{\mathrm{B}}\right.$ family). The total average density of nanostructures, $\mathrm{QD}_{\mathrm{A}}$ and $\mathrm{QD}_{\mathrm{B}}$, is the same as the initial nanoholes density, $\rho=2 \times 10^{8} \mathrm{~cm}^{-2}$.

Figure 2(b) corresponds to the deposition of 1.7 ML of InAs, the critical value for QD formation on a flat substrate. A higher density of QD $\left(\rho=2 \times 10^{9} \mathrm{~cm}^{-2}\right)$ is observed, involving QD nucleation both inside and outside the nanoholes. The QD formed at the nanoholes have typically diameters $\Phi=55.8 \pm 1.5 \mathrm{~nm}$ and height $h=20.3 \pm 1.0 \mathrm{~nm}$ referred to the flat surface plane while those nucleated surrounding the nanoholes show a broader size distribution with mean values of $\Phi=36.2 \pm 5.9 \mathrm{~nm}$ and $h=10.3 \pm 2.9 \mathrm{~nm}$.

Figure 2(c) shows the normalized nanostructures PL emission at excitation power of $0.5 \mathrm{~mW}$ for $1.1,1.3,1.5$, and 1.7 ML (blue, green, red, and black lines, respectively) of InAs deposited over the nanoholes template. In the case of 1.1 ML of InAs a single and broad peak [full width at half maximum $(\mathrm{FWHM})=47 \mathrm{meV}]$ centered at $1.385 \mathrm{eV}$ is observed. For 1.3 ML of InAs two emission peaks are obtained at $1.25 \mathrm{eV}(\mathrm{FWHM}=33 \mathrm{meV})$ and $1.379 \mathrm{eV}$ (FWHM $=31 \mathrm{meV}$ ). Two emission peaks are also obtained at $1.19 \mathrm{eV}$
$1.3 \mathrm{ML} \operatorname{lnAs}$
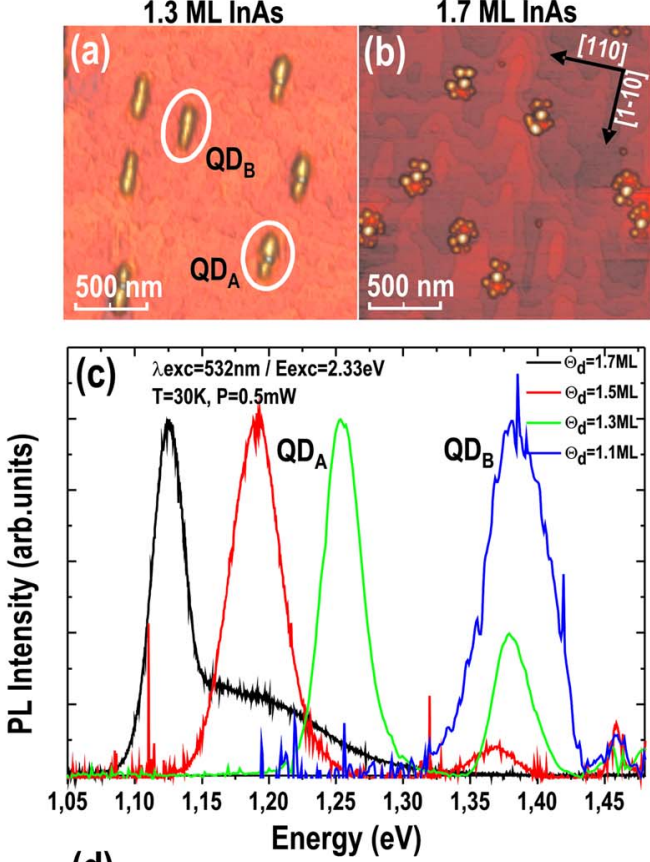

(d)

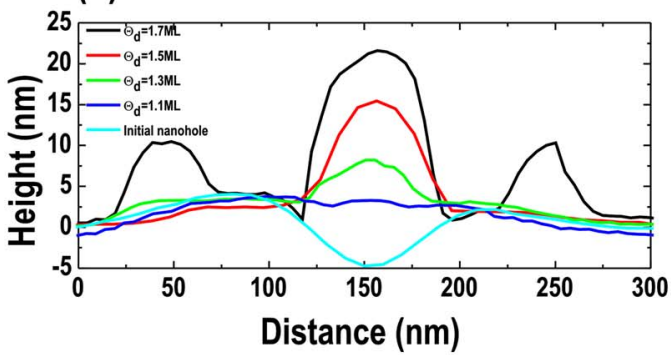

FIG. 2. (Color online) (a) $2 \times 2 \mu \mathrm{m}^{2}$ AFM micrograph showing the nanoholes template after growing $1.3 \mathrm{ML}$ of InAs. (b) $2 \times 2 \mu \mathrm{m}^{2} \mathrm{AFM}$ micrograph showing the nanoholes template after growing $1.7 \mathrm{ML}$ of InAs (c) PL spectra after growing 1.1, 1.3, 1.5, and 1.7 ML of InAs on the nanoholes template. (d) AFM profiles showing the filling of the GaAs nanohole from the initial empty state to the nucleation of $1.1,1.3,1.5$, and 1.7 ML of InAs.

$(\mathrm{FWHM}=44 \mathrm{meV})$ and $1.367 \mathrm{eV}(\mathrm{FWHM}=31 \mathrm{meV})$ for 1.5 ML of InAs deposited.

In the case of 1.1 ML of InAs, the broad PL emission observed would reflect the broad size distribution of initial nanoholes. However, a direct correlation between the size of the nanoholes and QD is not possible as no protruding QD were observed (AFM results not shown). On the other hand, according to the previous topographic images [Fig. 2(a)], the pair of PL peaks observed in the spectra for 1.3 and 1.5 ML of InAs would, respectively, correspond to the emission of $\mathrm{QD}_{\mathrm{B}}$ (peak at higher energies) and $\mathrm{QD}_{\mathrm{A}}$ (peak at lower energies).

For 1.7 ML of InAs deposited, we also observe two main PL peaks at 1.12 and $1.18 \mathrm{eV}(\mathrm{FWHM}=27$ and $117 \mathrm{meV}$, respectively). According to the AFM image shown in Fig. 2(b) and the QD size distributions obtained, they can be ascribed to QD formed inside and outside of the nanoholes. In this case, all the QDs in the nanoholes protrude, $\mathrm{QD}_{\mathrm{A}}$ type, as the filling of the nanoholes is large enough.

Thus, focusing on the situation where QD are formed at the nanoholes, $\mathrm{QD}_{\mathrm{A}}$ and $\mathrm{QD}_{\mathrm{B}}$, we observe a clear dependence on the emission energy of the two QD families with the amount of InAs material deposited. The close correlation between PL results and size of the QD is further illustrated in 


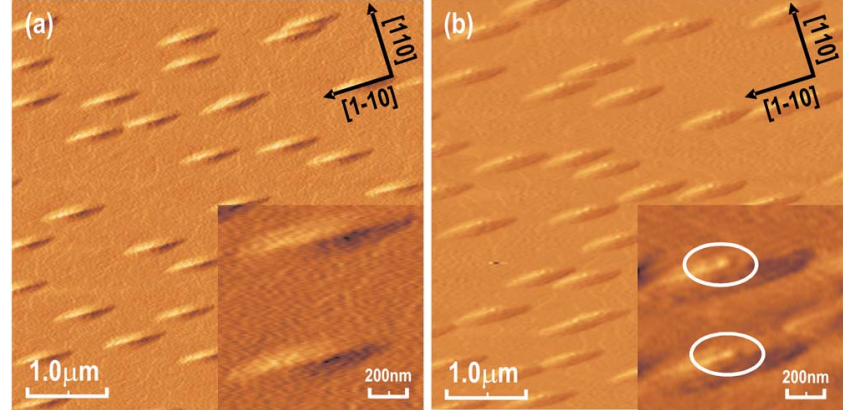

FIG. 3. (Color online) (a) $5 \times 5 \mu \mathrm{m}^{2}$ derivative AFM micrograph showing the surface after capping the InAs nanostructures with a $25 \mathrm{~nm}$ thick GaAs buffer layer. The inset shows a detailed $1 \times 1 \mu \mathrm{m}^{2}$ zoom of this image. (b) $5 \times 5 \mu \mathrm{m}^{2}$ derivative AFM micrograph showing the surface in (a) after the growth of 1.4 ML of InAs. The inset shows a detailed $1 \times 1 \mu \mathrm{m}^{2}$ zoom of this image. White circles surround the InAs nucleus formed on top of the mounds.

Fig. 2(d), showing the height profiles across the QD formed at the nanoholes with emission at lower energies $\left(\mathrm{QD}_{\mathrm{A}}\right.$ corresponding to $1.3,1.5$, and $1.7 \mathrm{ML}$ of InAs). The profiles of a QD formed at a nanohole in the case of 1.1 ML $\left(\mathrm{QD}_{\mathrm{B}}\right)$ and of an initial nanohole are also represented for comparison. In the case of 1.7 ML of InAs deposited the profile of QD surrounding the original nanohole is also shown. We observe that these QDs are slightly smaller than $\mathrm{QD}_{\mathrm{A}}$ corresponding to $1.3 \mathrm{ML}$ of InAs (measured from bottom of the nanohole). In spite of that, their PL emission peak is centered at lower energy. We want to notice that during InAs deposition, both In and $\mathrm{Ga}$ adatoms are filling the nanoholes. ${ }^{9}$ Accordingly, $\mathrm{QD}_{\mathrm{A}}$ formed at the nanoholes would have a greater Ga content than those formed around the nanoholes. A qualitative idea of the difference in composition of both kinds of QD can be extracted from the PL peak energy. Assuming for simplicity that QDs formed around the nanoholes are 100\% InAs and using a linear interpolation of the band gap energy, a Ga content of $20 \%$ for $\mathrm{QD}_{\mathrm{A}}$ (formed at the nanoholes) is obtained.

When these QD are capped, we observe (AFM images not shown) that the resulting surface shows mounds all over it. If these mounds had its origin in the buried strained nanostructures, the propagation of strain through the cap layer would provide preferential nucleation sites for the growth of the next InAs nanostructures. In order to prove this assumption, we have grown samples with $25 \mathrm{~nm}$ thick GaAs cap layers where the strain effect would be much larger. The AFM image in Fig. 3(a) corresponds to the surface of a 25 $\mathrm{nm}$ thick GaAs layer covering 1.4 ML of InAs deposited over the nanoholes template. We observe mounds elongated along the $[1 \overline{1} 0]$ direction that presents the same density obtained for the initial buried nanostructures $\rho=2 \times 10^{8} \mathrm{~cm}^{2}$ [Fig. 1(a)]. Their typical dimensions are $700 \mathrm{~nm}$ in length, $150 \mathrm{~nm}$ in width, and $7 \mathrm{~nm}$ in height. Their origin could be related to the shape evolution of the already elongated structures formed at the nanoholes during the earliest grown monolayers of the cap layer. ${ }^{12}$ We have observed that this surface morphology is propagated for cap layer thickness up to $100 \mathrm{~nm}$. However, we would expect that these mounds will remain for thickness as large as desired using the appro- priate growth conditions, as those provided by atomic layer MBE growth mode. ${ }^{13}$

Assuming that these mounds are located on the strained buried nanostructures, the InAs based nanostructures formed on this surface would preferentially nucleate on top of the mounds. ${ }^{14}$ Figure 3(b) shows the AFM image of an identical sample to the previous one [Fig. 3(a)] but continuing the growth process with the deposition of 1.4 ML of InAs. Notice that the amount of InAs deposited is far from the critical for QD formation (1.7 ML) on a flat surface. This means that we would not expect QD formation unless InAs growth is enhanced at preferential sites of the surface. It can be observed in the figure that an incipient InAs nanostructure appears on top of each mound (white circles) confirming their direct correspondence with the buried nanostructures.

In conclusion, we have extended the droplet epitaxy technique for growing $\operatorname{In}(\mathrm{Ga}) \mathrm{As}$ QD which emission wavelength can be tuned in a wide range maintaining a total density of nanostructures lower than $2 \times 10^{8} \mathrm{~cm}^{-2}$. Moreover, the surface obtained after the capping growth process presents mounds that are directly correlated with the buried nanostructures as proved by the posterior preferential growth of InAs on top of them. These results are highly relevant for quantum optic devices fabrication where low density nanostructures with control in energy emission and exact surface localization are optimal requirements.

The authors acknowledge the financial support by Spanish MEC and CAM through Project Nos. TEC-2005-05781C03-01/-03 and NAN2004-09109-C04-01/-03, by Consolider Grant No. QOIT CSD2006-0019 and S-505/ESP/ 000200 .

${ }^{1}$ R. M. Stevenson, R. J. Young, P. Atkinson, K. Cooper, D. A. Ritchie, and A. J. Shields, Nature (London) 439, 179 (2006).

${ }^{2}$ K. Hennessy, A. Badolato, M. Wigner, D. Gerace, M. Atatüre, S. Gulde, D. Fält, E. L. Hu, and A. Imamoglu, Nature (London) 445, 896 (2007).

${ }^{3}$ P. M. Intallura, M. B. Ward, O. Z. Karimov, Z. L. Yuan, P. See, A. J. Shields, P. Atkinson, and D. A. Ritchie, Appl. Phys. Lett. 91, 161103 (2007).

${ }^{4}$ D. Guimard, H. Lee, M. Nishioka, and Y. Arakawa, Appl. Phys. Lett. 92, 163101 (2008).

${ }^{5}$ J. Martín-Sánchez, Y. González, L. González, M. Tello, R. García, D. Granados, J. M. García, and F. Briones, J. Cryst. Growth 284, 313 (2005)

${ }^{6}$ S. Kiravittaya, M. Benyoucef, R. Zapf-Gottwick, A. Rastelli, and O. G. Schmidt, Appl. Phys. Lett. 89, 233102 (2006)

${ }^{7}$ P. Atkinson, M. B. Ward, S. P. Bremner, D. Anderson, T. Farrow, G. A. C. Jones, A. J. Shields, and D. A. Ritchie, Jpn. J. Appl. Phys., Part 145 , 2519 (2006).

${ }^{8}$ P. Alonso-González, L. González, Y. González, D. Fuster, I. FernándezMartínez, J. Martín-Sánchez, and L. Abelmann, Nanotechnology 18, 355302 (2007).

${ }^{9}$ R. Songmuang, S. Kiravittaya, and O. G. Schmidt, Appl. Phys. Lett. 82, 2892 (2003)

${ }^{10}$ Zh. M. Wang, B. L. Liang, K. A. Sablon, and G. J. Salamo, Appl. Phys. Lett. 90, 113120 (2007).

${ }^{11}$ P. Alonso-González, B. Alén, D. Fuster, Y. González, L. González, and J. Martínez-Pastor, Appl. Phys. Lett. 91, 163104 (2007).

${ }^{12}$ G. Costantini, A. Rastelli, C. Manzano, P. Acosta-Diaz, R. Songmuang, G. Katsaros, O. G. Schmidt, and K. Kern, Phys. Rev. Lett. 96, 226106 (2006).

${ }^{13}$ F. Briones, L. González, and A. Ruiz, Appl. Phys. A: Solids Surf. A49, 729 (1989).

${ }^{14}$ B. Yang, F. Liu, and M. G. Lagally, Phys. Rev. Lett. 92, 025502 (2004). 